\title{
PADRÕES DE TRANSPORTE DE SEDIMENTOS BASEADO EM TRÊS PROGRAMAS GERADORES DE VETORES DE TENDÊNCIAS DE TRANSPORTE A PARTIR DE PARÂMETROS GRANULOMÉTRICOS NA PORÇÃO SUL DO DELTA DE DESEMBOCADURA DO COMPLEXO ESTUARINO DE PARANAGUÁ - SUL DO BRASIL

\author{
NET SEDIMENTS TRANSPORT PATHS BASED ON THREE GRAIN-SIZE \\ TRENDS ANALYSIS PROGRAMS IN THE SOUTH SECTOR OF THE
} PARANAGUÁ ESTUARINE COMPLEX OUTLET DELTA - SOUTH BRAZIL
}

\author{
Fernando Alvim Veiga ${ }^{1}$ \\ Rodolfo José Angulo² \\ Eduardo Marone ${ }^{3}$ \\ Frederico Pereira Brandini ${ }^{3}$ \\ Carlos Roberto Soares ${ }^{4}$
}

\begin{abstract}
RESUMO
Este trabalho apresenta os resultados da aplicação dos programas de geração de vetores de tendência de transporte de sedimentos GSTA, GSTAST e TRANSVEC, baseados em parâmetros granulométricos (média granulométrica, desvio padrão e assimetria) de sedimentos da porção sul do delta de desembocadura do Complexo Estuarino de Paranaguá. O transporte preferencial na área estudada dá-se de sudoeste para nordeste, regido pela passagem de sistemas frontais provenientes de sul que transferem grandes quantidades de energia ao sistema, havendo o empilhamento destes sedimentos causado pelo efeito "molhe hidráulico" das correntes predominantes de vazante na desembocadura sul do estuário, tornando-a mais pronunciada que a parte norte. Este fenômeno possibilita o desenvolvimento de um extenso banco de areia, denominado banco da Galheta, com profundidades variando entre 1,5 a 5,0 metros na região, onde as ondas quebram causando o transporte
\end{abstract}

\footnotetext{
1 Universidade Federal do Paraná, Departamento de Geologia, Pós-graduação em Geologia. e.mail:ferveiga@ufpr.br 2 Universidade Federal do Paraná, Departamento de Geologia, Pesquisador CNPq.

3 Universidade Federal do Paraná, Centro de Estudos do Mar, Pesquisador CNPq.

4 Universidade Federal do Paraná, Centro de Estudos do Mar.
} 
VEIGA, F. A. et al. Padrões de transporte de sedimentos baseado em três...

em direção à costa, evidenciado por esporões que migram embocadura adentro. Após a análise dos resultados, observa-se que os vetores gerados pelo programa GSTA encaixam-se perfeitamente na batimetria da área. Estão de acordo com o esperado na parte sul e central da área, mas não parecem bons nos setores NE e NW da área. O programa TRANSVEC identifica muito bem o direcionamento do transporte para o canal que margeia o delta e também uma tendência do canal marginal de exportar sedimentos durante maré vazante na parte NW da área. Os vetores indicam transporte preferencial para SW, o que contraria todas as observações para a área em questão. O programa que melhor satisfaz as tendências esperadas é o GSTAST. O teste de significância para os vetores, bem como a geração de vetores adicionais empíricos que são utilizados no processo de filtragem do GSTAST, funcionou a contento neste caso em particular. Os vetores a leste da área refletem o transporte de material pelas ondas quebrando no banco da Galheta, os vetores centrais mostram o empilhamento da deriva de sul para norte sobre o delta de desembocadura e os vetores a oeste da área refletem a deflexão da deriva litorânea próximo à súbita queda na profundidade causada pela presença do delta.

Palavras-chave: transporte de sedimentos; tendências de transporte; vetores de tendência.

\begin{abstract}
This work presents the results of GSTA, GSTAST and TRANSVEC grain size trend analysis programs based in mean size, standard deviation and skewness parameters of bottom sediments at the south portion of the Paranaguá Estuarine Complex Outlet Delta. The longshore sediment transport is from southwest to northeast in the area, ruled by episodically high-energy frontal systems events. Consequently, the sediments are trapped by the hydraulic dam formed by tidal currents and the south part of the outlet is quite large that the north portion. Thus, an extended breaking wave field from 1.5 to 5 meters water depth named Galheta Bank is formed. Currents and wave action in this bank sediments cause spit formation that moves landward into the bay mouth. The result suggests that the trends generated by GSTA program fits with the bathymetry. They are according to the expectations in the southern and center sector of the area, but they are not in the NE and NW sectors. The TRANSVEC program identifies the transport path to the marginal channel of the delta and the tendency of this channel to export sediments during strong ebb tide currents in the NW sector, but the vectors also indicate SW preferential transport that contradicts field observations. The most consistent result was obtained by GSTAST program that generates empiric data and a significant test with $95 \%$ confidence before validating or not the resultant vectors. The eastern vectors reflect wave sediment transport on the Galheta bank, central vectors show deposition by longshore drift over the bay mouth delta and the west vectors are according with the deflection of the longshore currents caused by the sudden decrease of depth in the delta.

Key-words: sediment transport; transport trends; grain size trends.
\end{abstract}

\section{INTRODUÇÃO}

O significado e a aplicabilidade dos diversos parâmetros estatísticos que podem ser calculados a partir da análise granulométrica de um sedimento têm gerado discussões no meio científico. Nas últimas duas décadas vários autores como McLaren (1981); McLaren \& Bowles (1985); Gao \& Collins (1991, 1992); Le Roux (1994a, 1994b); Gao (1996); Pedreros et al. (1996); Chang et al. (2001), só para citar alguns, têm acordado que a média granulométrica, o grau de seleção e a assimetria do sedimento são os parâmetros mais importantes para a determinação da direção de transporte dos sedimentos em um ambiente deposicional. No entanto, devido à diversidade de fatores que podem variar no ambiente, as tentativas de elaborar modelos e programas para serem aplicados com segurança na natureza têm se mostrado polêmicas.
McLaren (1981) conclui que: 1) as características granulométricas de um depósito sedimentar dependem: da deposição seletiva do sedimento durante o transporte, da total deposição do sedimento transportado, além das características do depósito fonte. As relativas mudanças ou tendências destas características, definidas pela média granulométrica, seleção e assimetria, podem ser usadas para determinar fontes e depósitos que têm participação nos processos e, por inferência, a direção preferencial do transporte do sedimento. 2) Exceções nestas tendências podem ocorrer se houver mais de uma fonte de sedimento, se houver processos coesivos em sedimentos finos ou floculação durante a deposição e se o tamanho dos grãos alterar-se durante o transporte. 3) Somente a distribuição do tamanho dos grãos de um sedimento não pode ser utilizada para determinar com segurança o ambiente de deposição. 4) A análise 
VEIGA, F. A. et al. Padrões de transporte de sedimentos baseado em três...

das tendências dos parâmetros granulométricos pode ser usada para determinar: a) prováveis relações entre os ambientes de deposição; b) os processos mais importantes que operam nos sub-ambientes; c) a direção de transporte de sedimentos; e d) os ambientes de deposição.

Em síntese, baseando-se em um sedimento com distribuição hipotética e assumindo que grãos mais leves apresentam maior probabilidade de serem erodidos e transportados do que grãos pesados McLaren (1981) afirma que: 1) durante o transporte os sedimentos tornam-se mais finos, melhor selecionados e com assimetria mais negativa do que o sedimento fonte; 2) podem também se tornar mais grossos, melhor selecionados e com assimetria mais positiva ; 3) Em depósitos sucessivos, os sedimentos tendem a ficar mais finos ou mais grossos, mas a seleção tende a melhorar e a assimetria a ficar mais positiva.

Mais tarde, McLaren \& Bowles (1985) estabelecem um modelo relacionando os caminhos do transporte de sedimentos a variações espaciais, considerando a média granulométrica $(\mu)$, um coeficiente do grau de seleção $\left(\sigma^{2}\right)$ e a assimetria (Sk), desenvolvendo um tratamento estatístico dos dados para gerar um modelo em uma dimensão das tendências de transporte do sedimento em um ambiente no qual seria possível distinguir dois padrões principais de direção de transporte efetivo na natureza. No sentido do transporte, os sedimentos se tornariam mais grossos, melhor selecionados e com assimetria mais positiva, ou mais finos, melhor selecionados e com assimetria mais negativa:

$$
\begin{aligned}
& \sigma_{1}^{2}<\sigma_{2}^{2}, \mu_{1}<\mu_{2} \& S k_{1}<S k_{2} \\
& \sigma_{1}^{2}>\sigma_{2}^{2}, \mu_{1}<\mu_{2} \& S k_{1}>S k_{2}
\end{aligned}
$$

sendo os subscritos 1 e 2 os locais de amostragem: 2 estando ao longo da direção de transporte de 1 e os valores em unidades $\Phi$.

Gao \& Collins (1991) fazem uma análise crítica do trabalho de McLaren \& Bowles (1985), afirmando que, apesar dos casos 1 e 2 acima descritos serem dominantes, outros casos podem ocorrer e causar distorções no método. Consideram outros dois tipos de tendência possíveis no transporte de sedimentos:

$$
\begin{aligned}
& \sigma_{1}^{2}>\sigma_{2}^{2}, \mu_{1}>\mu_{2} \& S k_{1}>S k_{2} \\
& \sigma_{1}^{2}>\sigma_{2}^{2}, \mu_{1}<\mu_{2} \& S k_{1}<S k_{2}
\end{aligned}
$$

Estes autores introduzem técnicas de filtragem semiquantitativas e testes de significância para todos os casos (Gao \& Collins 1992, 1994), o que resulta em um modelo em duas dimensões que gera padrões de vetores residuais de transporte de sedimentos.
Le Roux (1994a) faz uma análise crítica dos resultados obtidos por Gao \& Collins (1992) encontrando vetores "enigmáticos" que poderiam resultar da escolha subconsciente de padrões de transporte de sedimentos tanto do tipo 1 como do tipo 2. Outro problema seria a definição vaga de estações de vizinhança que adviria quando se assume uma distância representativa do espaçamento entre os pontos amostrais. Além disto, Le Roux (1994a) afirma que definir vetores considerando apenas duas amostras de cada vez não caracterizaria uma técnica verdadeiramente bidimensional.

Le Roux (1994b) propõe então uma nova abordagem para estimar a tendência de transporte de sedimentos. Em vez de analisar dois pontos amostrais de cada vez, o autor propõe a análise em grupos de cinco pontos, um central e quatro estações satélites eqüidistantes e a $90^{\circ}$ uma da outra, o que eliminaria os problemas do método de Gao \& Collins (1992).

Vários autores aplicaram os diversos métodos descritos acima em diferentes ambientes deposicionais, cada um com suas particularidades. Algumas tentativas foram bem sucedidas como a de Pedreros et al. (1996), que aplicaram o método de Gao \& Collins (1992) para a determinação de padrões de transporte de sedimentos em uma região intermarés em uma praia da costa Atlântica, ao sul da França, com regime de macromarés com resultados positivos. As principais razões para o funcionamento do método seriam: 1) apenas uma unidade sedimentar bem delimitada foi amostrada; 2) a camada de mistura de sedimento pôde ser reconhecida e propriamente amostrada; 3) o impacto causado pela densidade das amostras pôde ser testado; 4) a taxa e a direção de transporte puderam ser investigadas com traçadores para confirmar as direções gerais dos padrões de transporte residual.

Gao (1996) publica o programa GSTA (Grain Size Trend Analysis) relacionando oito tipos básicos de tendência de transporte de sedimentos:

$$
\begin{aligned}
& \text { Tipo } 1-\sigma_{2} \leq \sigma_{1}, \mu_{2} \geq \mu_{1}, \mathrm{Sk}_{2} \leq \mathrm{Sk}_{1} \\
& \text { Tipo } 2-\sigma_{2} \leq \sigma_{1}, \mu_{2} \leq \mu_{1}, \mathrm{Sk}_{2} \leq \mathrm{Sk}_{1} \\
& \text { Tipo } 3-\sigma_{2} \leq \sigma_{1}, \mu_{2} \geq \mu_{1}, \mathrm{Sk}_{2} \geq \mathrm{Sk}_{1} \\
& \text { Tipo } 4-\sigma_{2} \leq \sigma_{1}, \mu_{2} \leq \mu_{1}, \mathrm{Sk}_{2} \leq \mathrm{Sk}_{1} \\
& \text { Tipo } 5-\sigma_{2} \geq \sigma_{1}, \mu_{2} \geq \mu_{1}, \mathrm{Sk}_{2} \leq \mathrm{Sk}_{1} \\
& \text { Tipo } 6-\sigma_{2} \geq \sigma_{1}, \mu_{2} \leq \mu_{1}, \mathrm{Sk}_{2} \geq \mathrm{Sk}_{1} \\
& \text { Tipo } 7-\sigma_{2} \geq \sigma_{1}, \mu_{2} \geq \mu_{1}, \mathrm{Sk}_{2} \geq \mathrm{Sk}_{1} \\
& \text { Tipo } 8-\sigma_{2} \geq \sigma_{1}, \mu_{2} \leq \mu_{1}, \mathrm{Sk}_{2} \leq \mathrm{Sk}_{1}
\end{aligned}
$$

Para seu estudo em particular, o referido autor indica os tipos 1 e 2 como os mais apropriados para análise de tendência de transporte de sedimentos e, para validar o emprego do programa, frisa a necessidade da 
VEIGA, F. A. et al. Padrões de transporte de sedimentos baseado em três...

malha amostral ser compatível com o ambiente deposicional estudado.

Ainda segundo Gao (1996), os processos analíticos para a determinação das tendências consideram os seguintes passos:

1) Os parâmetros granulométricos de cada estação são comparados com os das estações vizinhas. Se as tendências de tipos 1 ou 2 ocorrerem entre uma estação central e qualquer das estações vizinhas, um vetor de tendência adimensional é definido para a estação central. Para identificar a validade da estação vizinha, uma distância característica $\left(\boldsymbol{D}_{c r}\right)$, que representa a escala da malha amostral é especificada. Se a distância entre duas estações for menor que $D_{c r}$ estas serão consideradas vizinhas e os parâmetros granulométricos serão comparados.

2) Uma transformação matemática é então realizada para as estações de amostragem que obtiverem mais de um vetor de tendência de transporte identificados no passo (1), resultando em apenas um vetor de tendência para cada estação amostral.

3) Um filtro é então aplicado para reduzir algum ruído remanescente (qualquer vetor que não seja consistente, em termos de direção, com os padrões de transporte é considerado um ruído). Novamente, as estações vizinhas são identificadas pela distância característica $\left(\boldsymbol{D}_{c r}\right)$ para realizar a filtragem.

Chang et al. (2001) apresentam uma sugestão para o método de Gao (1996), incluindo a geração, a partir dos dados reais, de dados empíricos realocados randomicamente, para testar se os vetores residuais representam realmente as direções preferenciais de transporte de sedimentos. Os dados empíricos são então utilizados para gerar vetores residuais. Este experimento é repetido a fim de se obter séries de vetores residuais para cada estação e para grupo de dados, estabelecendo a freqüência de ocorrência dos vetores por estação. Em seguida é realizado um teste de significância do vetor de tendência de transporte com um intervalo de confiança de $95 \%$, que representaria uma probabilidade de uma em vinte de que o vetor residual identificado tenha chance de ocorrer. Estes procedimentos são apresentados no programa GSTAST (Grain- Size Trend Analysis with Significant Test), que combina o algoritmo proposto, apagando os vetores artificiais calculados pelo programa GSTA de Gao (1996).

Le Roux et al. (2002), baseado em seu modelo de uma estação amostral central e quatro satélites, propõem o programa TRANSVEC em plataforma EXCELVBA em que os dados são: 1) selecionados automaticamente com opção de escolher a distância máxima entre o ponto central e os satélites; 2) o peso de cada parâmetro granulométrico pode ser alterado; 3) considera os quatro tipos de tendência de transporte principais:

$$
\begin{aligned}
& \text { Tipo } 1-\sigma_{2} \geq \sigma_{1}, \mu_{2} \leq \mu_{1}, S_{2} \geq S k_{1} \\
& \text { Tipo } 2-\sigma_{2} \leq \sigma_{1}, \mu_{2} \leq \mu_{1}, S_{2} \leq S k_{1} \\
& \text { Tipo } 3-\sigma_{2} \leq \sigma_{1}, \mu_{2} \leq \mu_{1}, S_{2} \geq S k_{1} \\
& \text { Tipo } 4-\sigma_{2} \geq \sigma_{1}, \mu_{2} \leq \mu_{1}, S_{2} \leq \mathrm{Sk}_{1}
\end{aligned}
$$

podendo ser extraído o de maior magnitude ou analisálos todos separadamente; 4) pode-se plotar os vetores resultantes com uma filtragem de 20,40, 60 e $80 \%$ da magnitude maior; 5) uma homogeneização dos vetores resultantes pode ser feita; 6 ) a distribuição dos dados vetoriais é analisada utilizando-se o teste não paramétrico de Watson (1966) que verifica se a tendência é preferencial ou uniforme.

A área escolhida para testar os programas situase na porção sul do delta de desembocadura do Complexo Estuarino de Paranaguá (CEP), onde foram coletadas amostras de sedimento em 81 pontos (figuras 1 e 2). A área possui aproximadamente $612 \mathrm{~km}^{2} \mathrm{de}$ superfície líquida (Knoppers et al. 1987), sendo adjacente a uma extensa planície costeira primeiramente estudada por Bigarella (1946). Posteriormente, Maak (1968) descreveu as principais unidades geológicas da planície costeira e da geografia física da região. Um estudo detalhado da planície costeira paranaense foi realizado por Angulo (1992), que apresenta mapas dos ambientes de sedimentação e das unidades geomorfológicas em escala 1:50.000 e 1:100.000 e, com base em indicadores sedimentares e biológicos, tece considerações sobre as variações do nível relativo do mar (NRM) durante o Pleistoceno e o Holoceno. Segundo Angulo (1992), a planície costeira do estado do Paraná é composta majoritariamente por sedimentos quaternários depositados em ambientes continentais e marinhos. Os sedimentos continentais são constituídos por cascalhos com matriz areno-argilosa, localizados nas proximidades do sopé de serras, na forma de depósitos de leques, tálus e colúvios, associados às vertentes. Os sedimentos marinhos são depósitos de areias finas a muito finas, quartzosas e bem selecionadas, constituindo feixes de cordões litorâneos orientados paralelamente à linha de costa atual (SW-NE), que ocorrem na forma de terraços cujas proporções decrescem em direção ao oceano. Nas proximidades do Balneário de Pontal do Sul, a altura dos terraços varia de 1,8 a 4,6 m. Sedimentos paleo-estuarinos, arenosos 


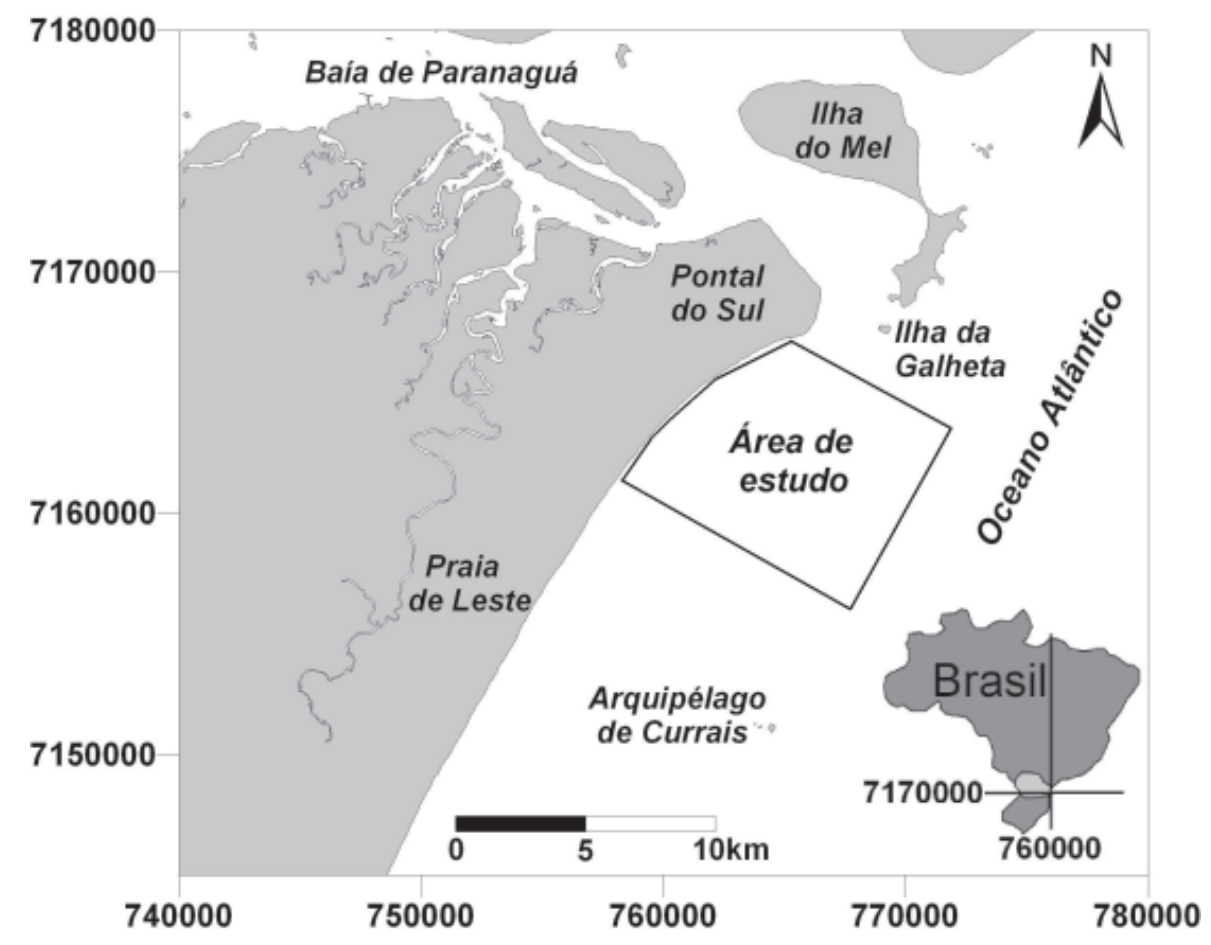

Figura 1 - Localização da área de estudo. Detail of the studied area.

e areno-argilosos, ocorrem em áreas baixas e associados ao fundo de antigos vales fluviais.

As feições do delta de desembocadura do CEP foram descritas por Angulo (1999). O autor observou esporões arenosos orientados em direção à terra presentes nas desembocaduras norte e sul do CEP, mostrando a eficiência da deriva longitudinal em transportar sedimentos estuário adentro. Além disso, formas de fundo indicando transporte de sedimentos para fora do estuário também são identificadas, evidenciando a complexidade do sistema em questão.

Existem dois sistemas de ondas preferenciais na área, de E/NE e SSE/SE (Portobras 1983). As ondas provenientes do quadrante SSE/SE são associadas à passagem de sistemas frontais que geram episódios de alta energia de ondas e parecem regular a dinâmica local. As ondas apresentam períodos entre 4 e 12 segundos e altura significativa entre 0,4 e 1,3 metros (Martins 2002). A amplitude de maré é de $1,5 \mathrm{~m}$, caracterizando regime de micromarés (Marone \& Camargo 1994) e a costa é dominada por tempestades (Veiga et al. 2004).

A presença dos portos de Paranaguá e Antonina gera a necessidade da dragagem periódica de um canal de acesso que corta o delta de desembocadura do CEP e, conseqüentemente, dados sobre transporte de sedimentos são muito importantes para a manutenção do canal. Angulo (1999) e Lamour (2000) fazem considerações empíricas sobre o transporte de sedimentos na região, mas uma tentativa de aplicar programas geradores de vetores de tendência de transporte nunca foi realizada na área e este é o objetivo principal deste trabalho.

A figura 2 apresenta o mapa da área estudada com as isóbatas e pontos amostrais.

\section{METODOLOGIA}

As amostras de sedimento de fundo foram obtidas com equipamento de busca a fundo tipo Petite Ponar, em 5 perfis transversais à linha de costa, predefinidos com distâncias entre estes de $2 \mathrm{~km}$, ao longo dos quais foram retiradas amostras a cada $600 \mathrm{~m}$, totalizando 81 amostras. As análises granulométricas foram processadas segundo os métodos descritos por Suguio (1973) para o peneiramento com peneiras a intervalos de 0,5 $\Phi$ e, para a pipetagem, intervalos de $1 \Phi$. Os resultados das análises granulométricas foram processados no programa SYSGRAN 2.4 (Camargo 1999) para a obtenção dos parâmetros estatísticos granulométricos segundo o método descrito por McCammon (1962) para a média granulométrica segundo a fórmula: 


$$
\sigma=\frac{\varnothing_{5}+\varnothing_{15}+\varnothing_{25}+\varnothing_{35}+\varnothing_{45}+\varnothing_{55}+\varnothing_{65}+\varnothing_{75}+\varnothing_{85}+\varnothing_{95}}{10}
$$

para o desvio padrão,

$$
\mu=\frac{\varnothing_{70}+\varnothing_{80}+\varnothing_{90}+\varnothing_{97}-\varnothing_{3}-\varnothing_{10}-\varnothing_{20}-\varnothing_{30}}{9,1}
$$

a assimetria é calculada pelo programa SYSGRAN 2.4, segundo a fórmula sugerida por Folk \& Ward (1957),

$$
S k=\frac{\emptyset_{16}+\varnothing_{84}-2 \varnothing_{50}}{2\left(\varnothing_{84}-\emptyset_{16}\right)}+\frac{\emptyset_{5}+\varnothing_{5}-2 \varnothing_{50}}{2\left(\varnothing_{95}-\emptyset_{5}\right)}
$$

Os parâmetros estatísticos (média granulométrica, assimetria e desvio padrão) foram processados nos programas GSTA (Gao 1996), GSTAST (Chang et al. 2001) e TRANSVEC (Le Roux et al. 2002) para a obtenção dos vetores de transporte de sedimentos. A amostra de número 42 foi considerada como sedimento palimpséstico (Veiga \& Angulo 2003) e os parâmetros estatísticos substituídos pela média dos dados referentes a seus pontos vizinhos. Os vetores foram plotados com o auxílio do programa SURFER ${ }^{\circledR} 7.0$ da Golden Software Corporation, que requer o trabalho adicional de multiplicar as direções de vetores resultantes por (-1), pois gira os vetores positivos no sentido anti-horário para plotá-los.

\section{RESULTADOS}

A partir dos parâmetros granulométricos utilizados para gerar os vetores de tendência de transporte de sedimentos, foram gerados os mapas a seguir pelo método de interpolação linear - triangulação W, correspondentes à média granulométrica, seleção e assimetria respectivamente (figuras 3, 4 e 5)

A figura 6 apresenta os vetores de transporte de sedimentos gerados pelo programa GSTA (Gao 1996) para as 81 amostras com um fator $\boldsymbol{A}$ igual a 1 porque as coordenadas dos pontos são em UTM e uma distância característica de 2200 metros (a maior distância entre um ponto e seus vizinhos é 2000 metros).

A figura 7 apresenta os vetores de transporte de sedimentos validados pelo programa GSTAST (Chang et al. 2001), considerando-se um número de repetição de estações empíricas igual a 160, distância característica $\left(\boldsymbol{D}_{c r}\right)$ de 2200 metros e um valor de $\boldsymbol{t}$ (coeficiente para o intervalo de confiança de $95 \%$ ) igual a 1,645 , que significa um intervalo de confiança de $95 \%$, como sugerido pelos autores. Estes parâmetros resultaram na validação de 27 vetores dos 81 possíveis. Alterando-se o número de

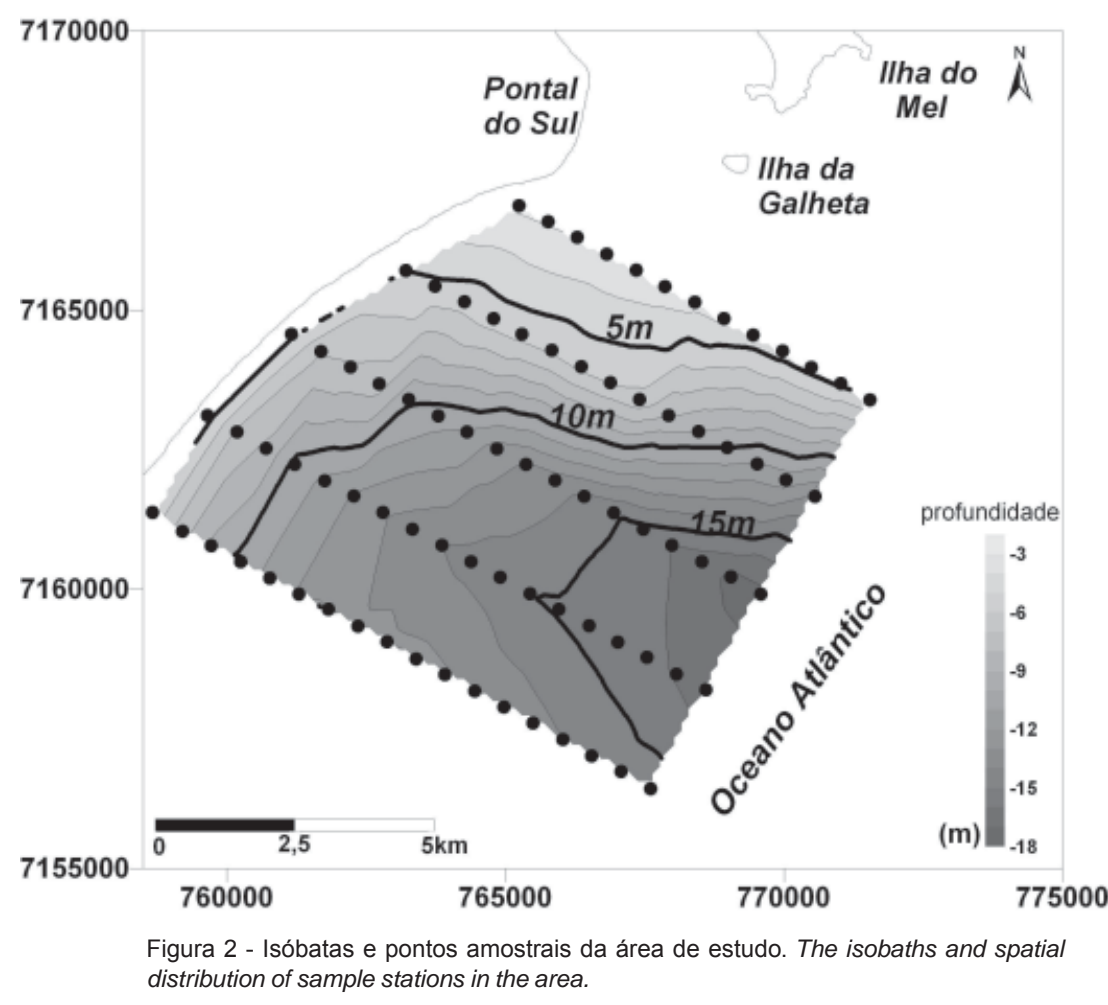


VEIGA, F. A. et al. Padrões de transporte de sedimentos baseado em três...

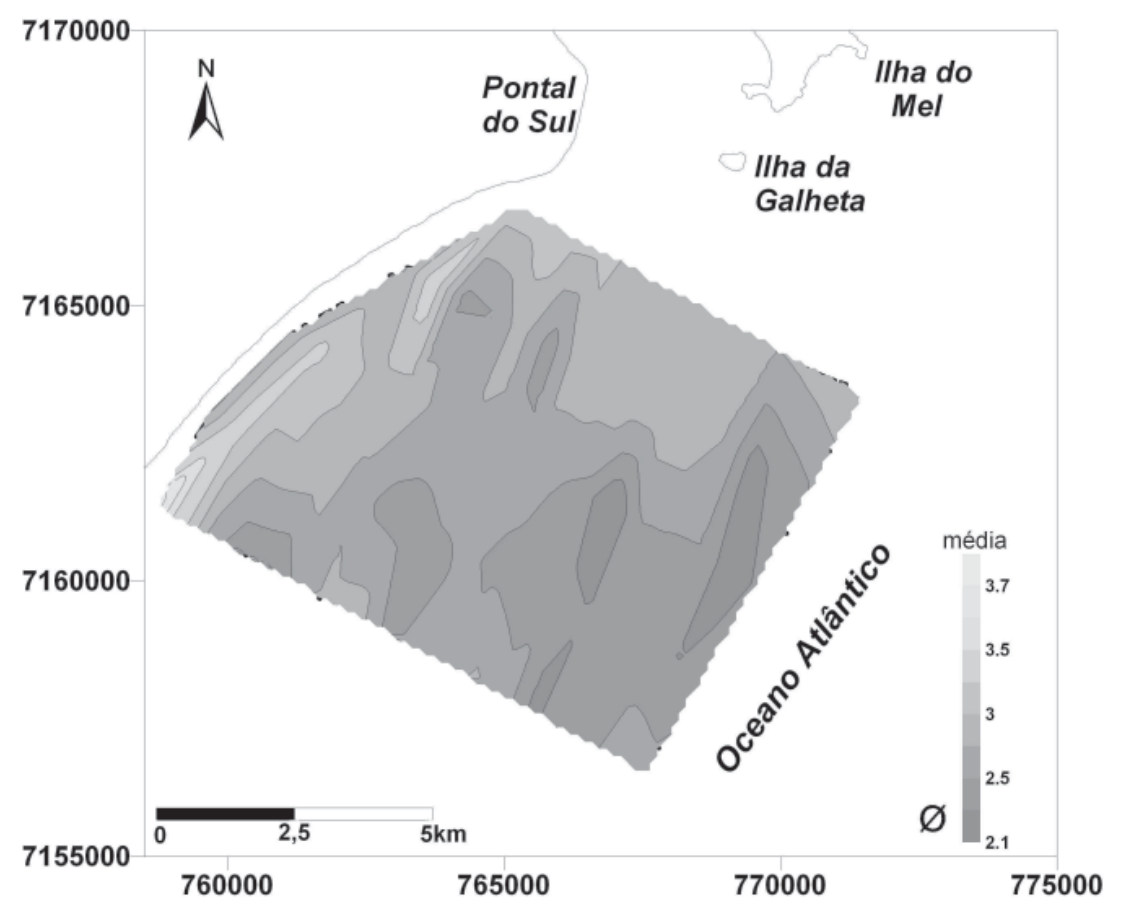

Figura 3 - Mapa de isolinhas para a média granulométrica do sedimento de fundo. The spatial distribution of mean grain size in the bottom sediments.

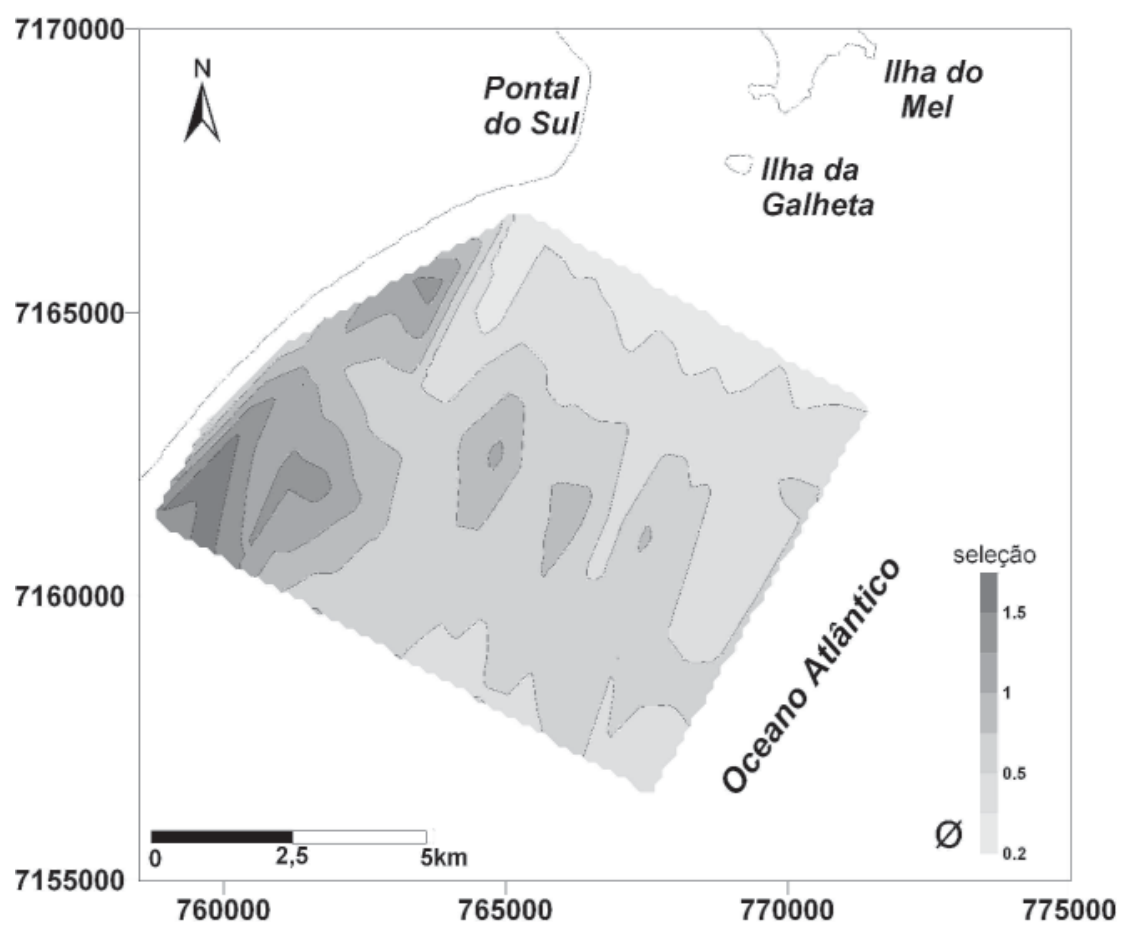

Figura 4 - Mapa de isolinhas para a seleção (desvio padrão) do sedimento de fundo. The spatial distribution of sorting in the bottom sediments. 
VEIGA, F. A. et al. Padrões de transporte de sedimentos baseado em três...

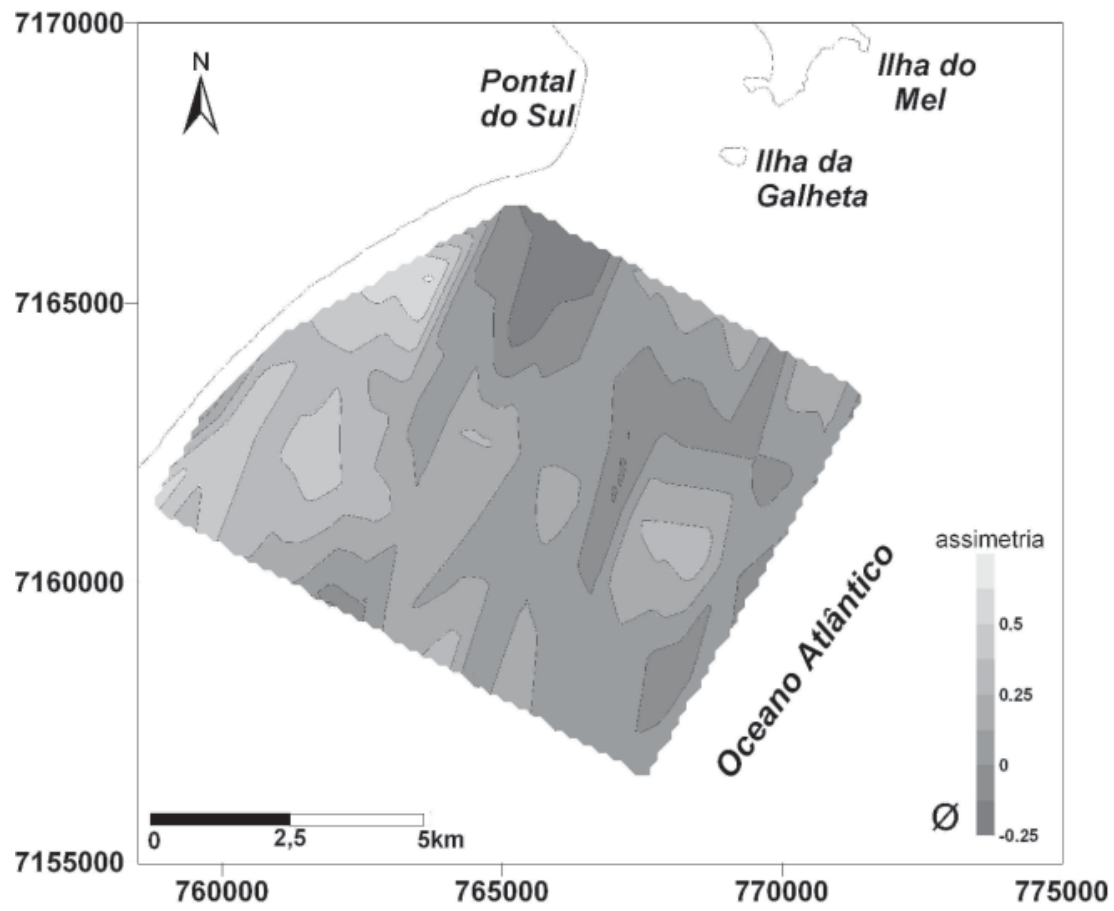

Figura 5 - Mapa de isolinhas para a assimetria do sedimento de fundo. The spatial distribution of skewness in the bottom sediments.

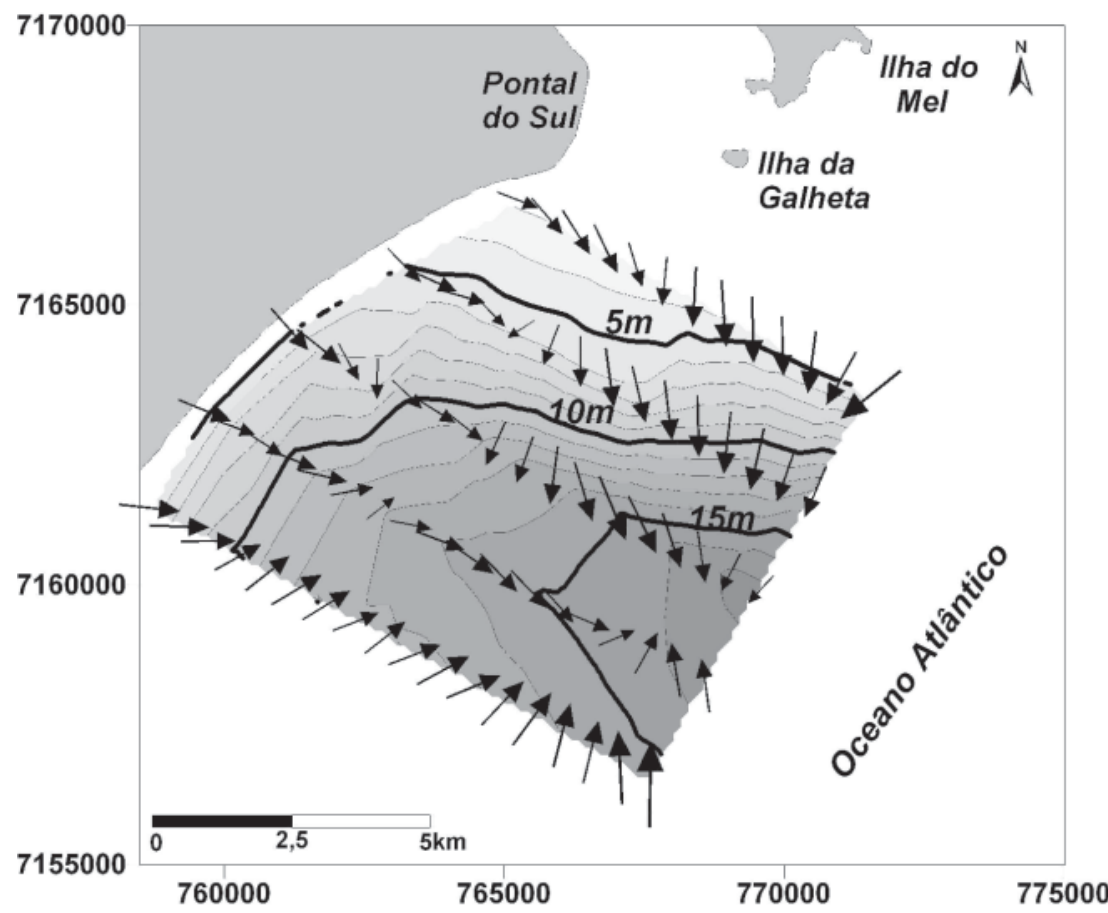

Figura 6 - Vetores de transporte de sedimentos gerados pelo programa GSTA (Gao 1996). Grain-size trends generated by GSTA program. 


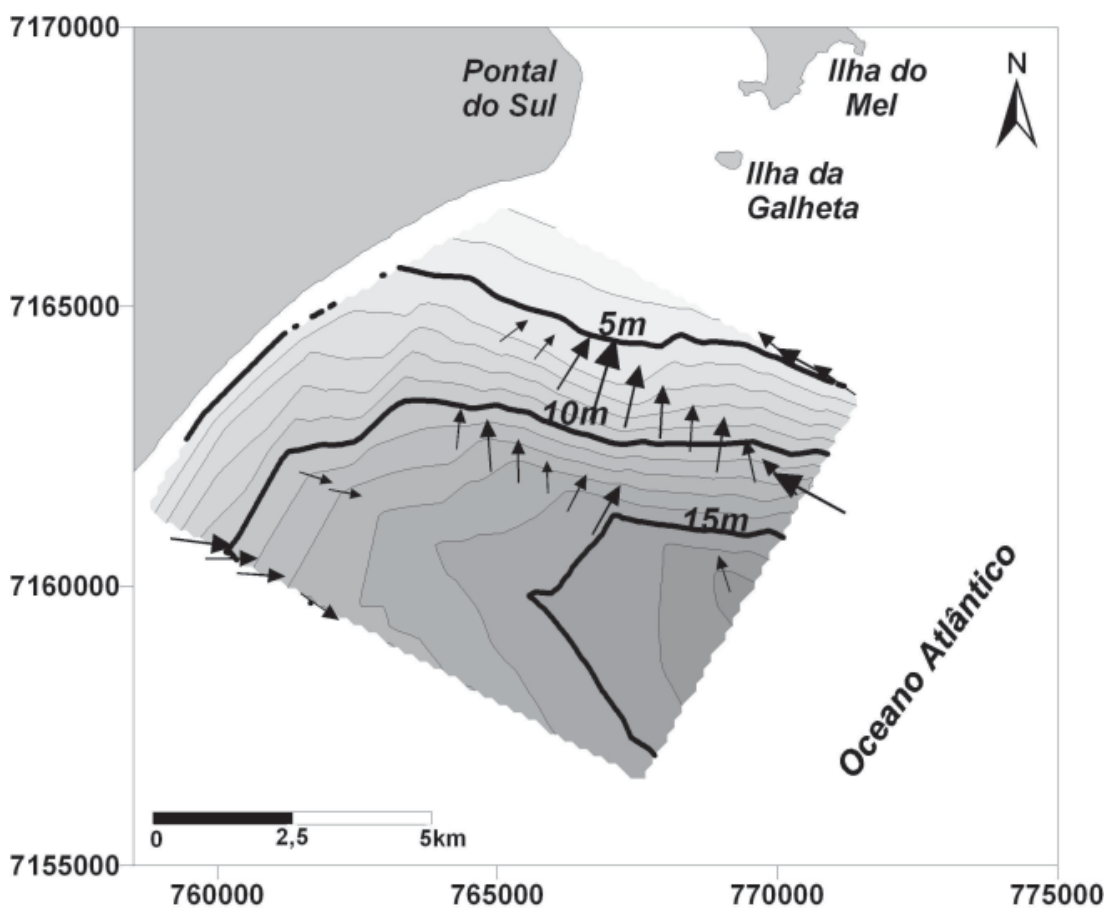

Figura 7 - Vetores de transporte de sedimentos validados pelo programa GSTAST (Chang et al. 2001). Grain-size trends generated by GSTAST program.

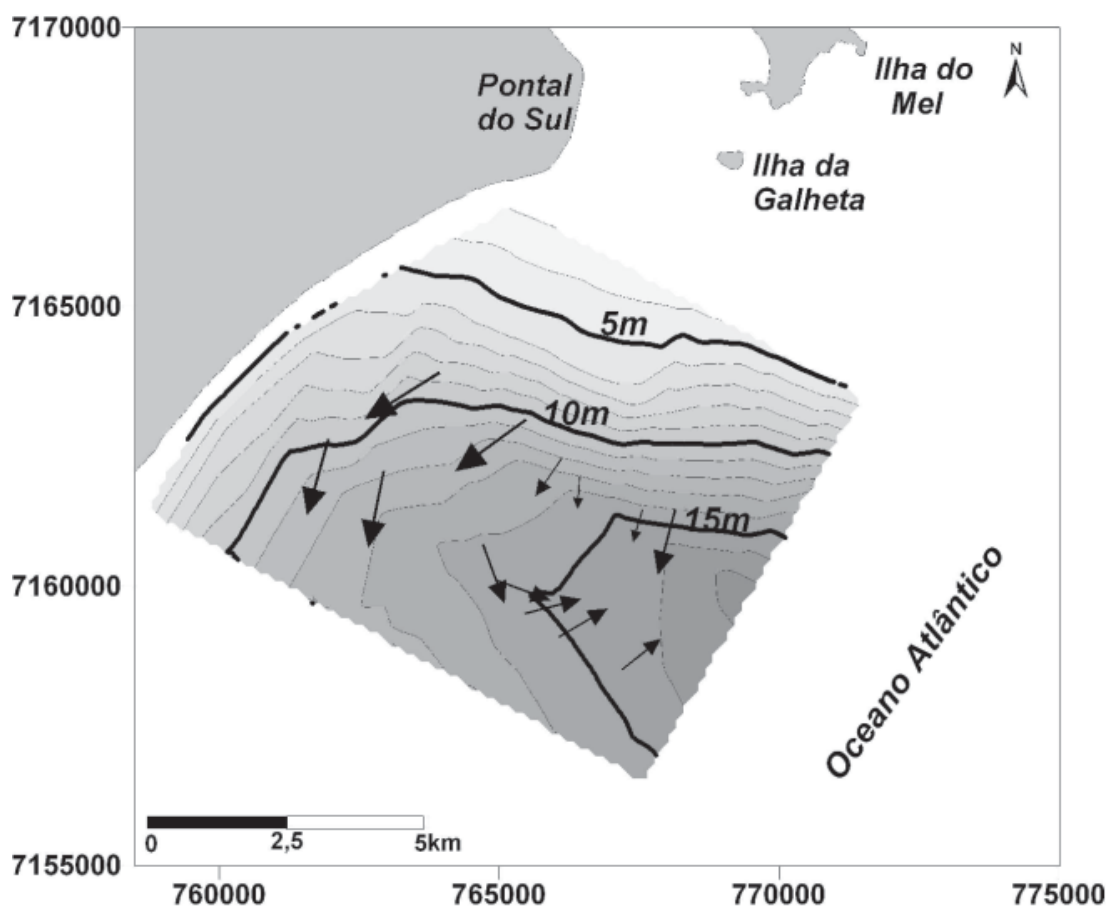

Figura 8 - Vetores de transporte de sedimentos validados pelo programa TRANSVEC (Le Roux 2002). Grain-size trends generated by TRANSVEC program. 
VEIGA, F. A. et al. Padrões de transporte de sedimentos baseado em três...

repetições para 100, o número de vetores possíveis foi de 26 (todos contidos nos 27 anteriores) e para 1000 e 5000 repetições, 25 vetores foram validados e contidos nos 27 primeiros. O valor de $\boldsymbol{t}$ foi mantido em 1,645 para todos os cálculos. Alterando-se o valor de $\boldsymbol{t}$ poder-se-ia conseguir a validação de mais vetores, porém, o teste de significância não atingiria o valor recomendado de $95 \%$ para o intervalo de confiança.

O programa TRANSVEC (Le Roux et al. 2002) foi utilizado para gerar os vetores válidos mostrados na figura 8 , considerando-se os quatro tipos principais de tendências de transporte. O raio de procura foi fixado em 3 vezes a média da distância entre as estações, peso para o cálculo estatístico de $33 \%$ para média granulométrica, $33 \%$ para seleção e $34 \%$ para assimetria. Alterando-se o parâmetro do raio de procura, não houve mudança significativa no resultado, bem como alterando o peso dos parâmetros estatísticos granulométricos, mesmo quando alterados significativamente. Foram gerados 18 vetores válidos após a execução do teste de Watson e 13 vetores após a operação de homogeneização (smooth vectors) que foram utilizados para plotagem.

\section{DISCUSSÃO}

O Complexo Estuarino de Paranaguá (CEP) foi mencionado como tal por Bigarella et al. (1978) e seu delta foi posteriormente considerado por Angulo (1992, 1995) como sistema deposicional de delta de desembocadura. Comparando fotografias aéreas da área de 1954 a 1980, Angulo (1993) descreve variações da linha de costa de dezenas a centenas de metros na região, sugerindo que os afloramentos rochosos da llha do Mel mantêm fixa a margem esquerda da desembocadura sul do CEP atualmente.

Angulo (1999) descreve esporões arenosos orientados em direção à terra presentes nas desembocaduras norte e sul do CEP, mostrando a eficiência da deriva longitudinal em transportar sedimentos estuário adentro. Além disso, formas de fundo indicando transporte de sedimentos para fora do estuário também são identificadas, evidenciando a complexidade do sistema em questão (figura 9). Este autor comenta que a porção sul do delta de desembocadura do CEP é a maior forma deposicional da costa paranaense, causando uma flexão na posição

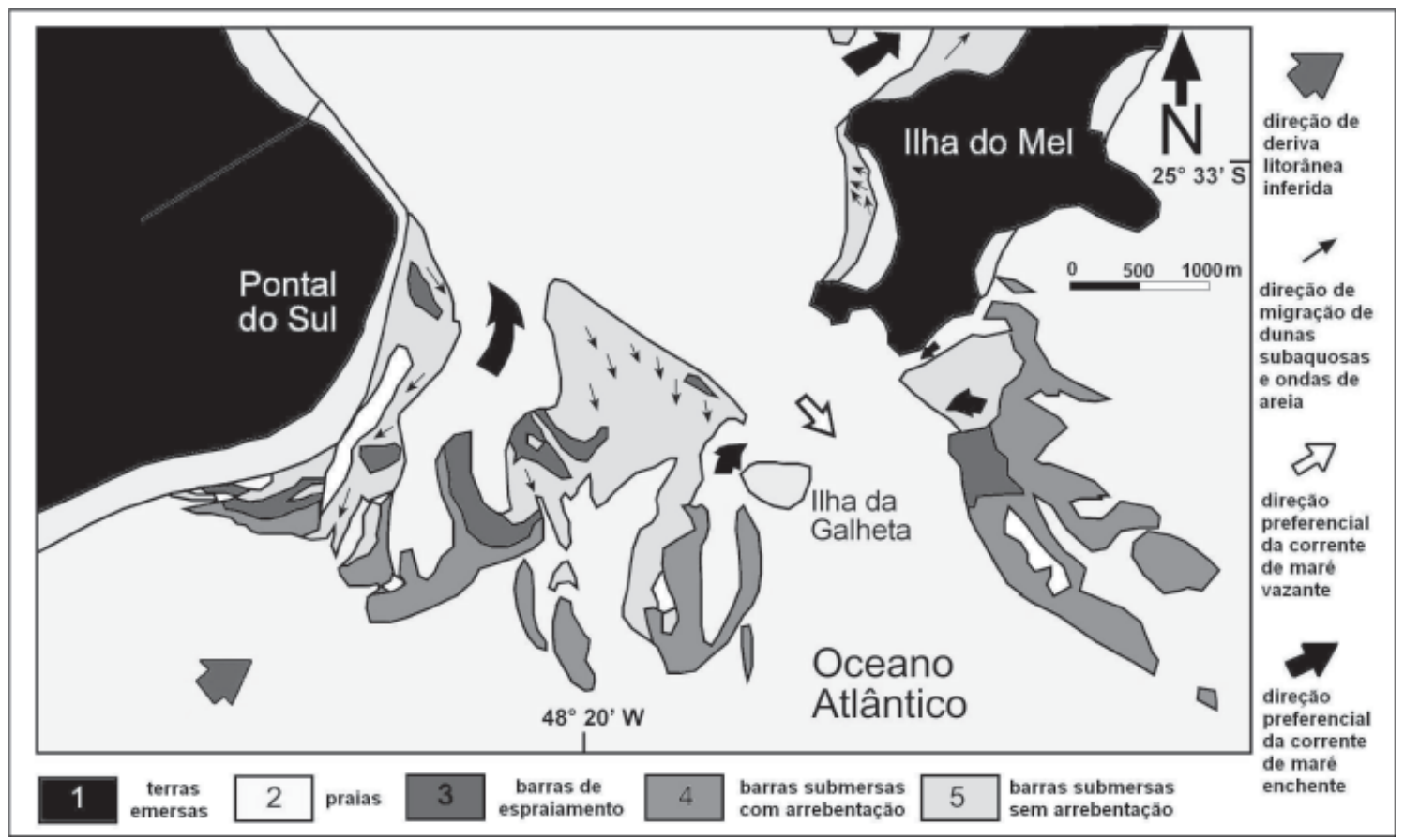

Figura 9 - Configuração da desembocadura sul do CEP (1) terras emersas; (2) praias; (3) barras de espraiamento; (4) barras submersas com arrebentação; (5) barras submersas sem arrebentação (modificado de Angulo 1999). Configuration of the southern outlet of the Paranaguá estuarine complex (1) land; (2) beaches; (3) swash bars; (4) breaking waves bars; (5) submerged bars without breaking waves. 


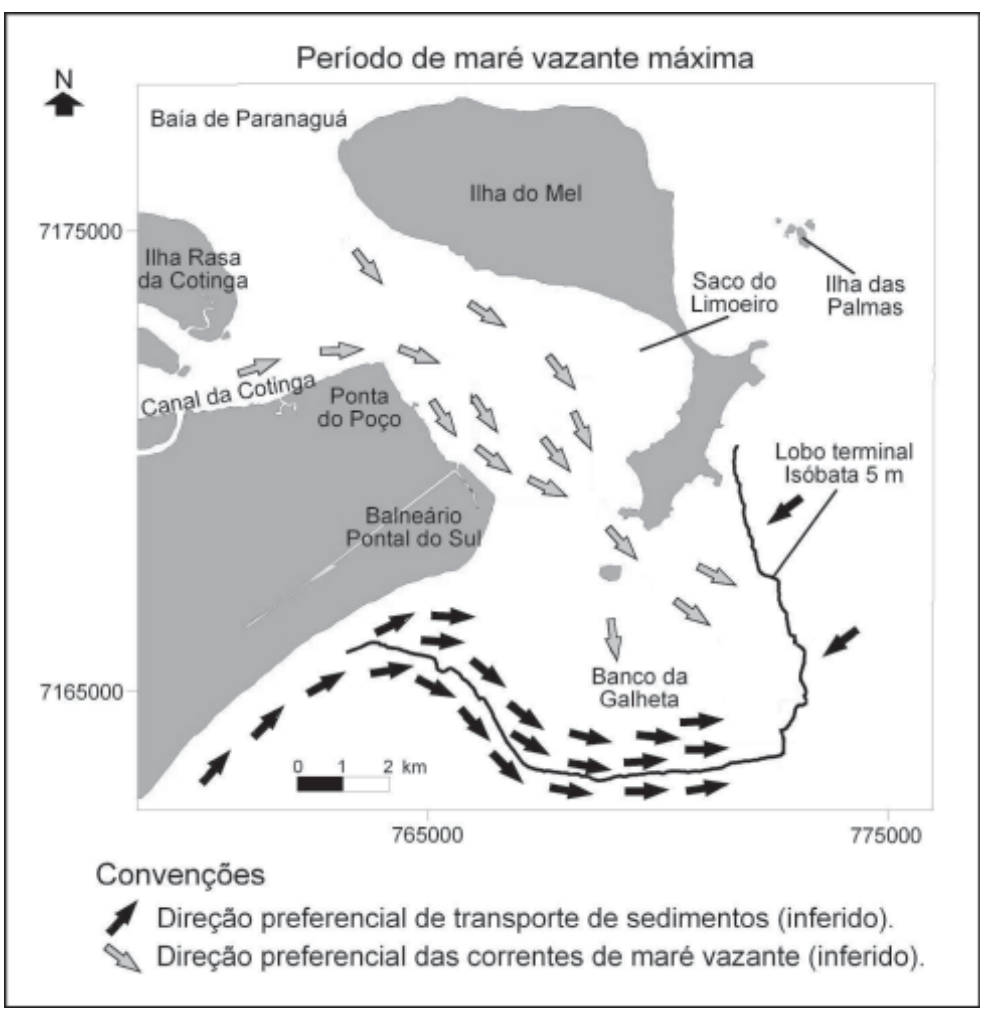

Figura 10 - Padrões inferidos de transporte de sedimentos para a porção sul do delta de desembocadura do Complexo Estuarino de Paranaguá (Lamour 2000). Inferred sediment transport patterns of the southern outlet of the Paranaguá estuarine complex.

da isóbata de 10 metros, que geralmente situa-se entre 1 e 3 km da costa, para 8 km em frente à costa do balneário de Pontal do Sul. Ainda segundo este autor, o maior desenvolvimento da porção sul do delta ocorre pela contribuição dos sedimentos trazidos pela deriva longitudinal de sul para norte, ali represados pelo efeito "barragem" ocasionado pelo fluxo das correntes de maré através do canal principal.

Lamour (2000), considerando características morfológicas e hidrodinâmicas do banco da galheta e o histórico das dragagens do canal artificial de acesso aos portos de Paranaguá e Antonina que corta o delta de desembocadura do Complexo Estuarino de Paranaguá, apresenta vetores adimensionais, inferidos a partir da observação de formas de fundo, para o transporte preferencial de sedimentos na porção sul do delta (figura 10).

O referido autor cita que a porção sul do delta de vazante é mais desenvolvida que a norte pelo efeito de "molhe hidráulico" das correntes de maré vazante que criam um maior espaço de acomodação para os sedimentos transportados de sul para norte pela deriva litorânea. Ainda segundo Lamour (2000), os sedimentos do banco da Galheta teriam sua origem nos sedimentos que se deslocam longitudinalmente à praia, entre a linha de costa e a isóbata de 10 metros. Esta afirmação é reforçada pela direção preferencial de deslocamento das correntes de deriva litorânea e pela presença de formas de leito na borda externa do banco. As correntes de maré enchente, que fluem pelo canal marginal do delta e as correntes de deriva litorânea entre Pontal do Sul e Ponta do Poço, contribuiriam para o transporte de sedimentos em direção ao interior do estuário.

É importante acrescentar que a ressuspensão e o transporte dos sedimentos causados pelos movimentos orbitais das ondas quebrando continuamente sobre as barras no Banco da Galheta são um agente transportador atuante e que seus efeitos devem estar registrados nos sedimentos deste ambiente deposicional. Há um empilhamento dos sedimentos transportados de sudoeste para nordeste sobre a desembocadura sul do Complexo Estuarino de Paranaguá que se encontra, atualmente, ancorada pelos afloramentos rochosos das Ilhas do Mel e da Galheta. Na figura 11, pode-se notar o extenso campo de ondas sobre o Banco da Galheta e o esporão (A) adentrando a embocadura da baía. 
VEIGA, F. A. et al. Padrões de transporte de sedimentos baseado em três...

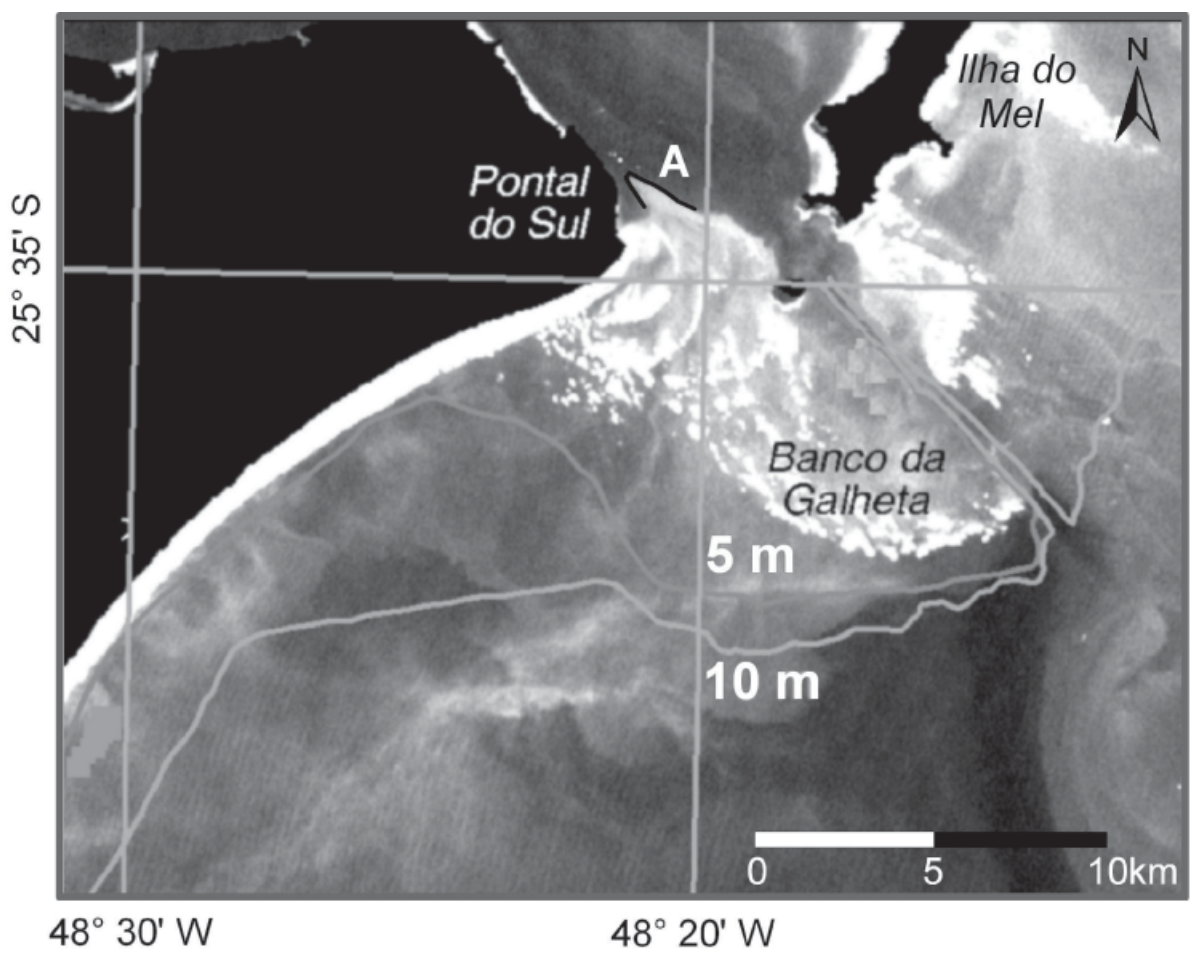

Figura 11 - Imagem de satélite da área de estudo evidenciando as proporções do Banco da Galheta (modificado de Noernberg 2001). Satellite image of the study area showing the Galheta bank amplitude.

\section{CONCLUSÕES}

Após a análise dos resultados obtidos com a aplicação dos programas de geração de vetores de tendências de transporte de material GSTA, GSTAST e TRANSVEC, observa-se que os vetores gerados pelo programa GSTA encaixam-se perfeitamente na batimetria da área. Estão de acordo com o esperado para a tendência de transporte de sedimentos, na parte sul e central da área, mas não parecem bons nos setores NE e NW da área.

Foram adicionados aos dados 18 estações a sul da área. Os vetores que compunham a linha que antes era a borda da área mudaram de configuração e representaram os vetores diferentemente. A geração de vetores empíricos fora da área em pontos em que não há dados disponíveis causa problemas no caso estudado. O programa TRANSVEC identifica muito bem o direcionamento do transporte para o canal que margeia o delta e também uma tendência do canal marginal de exportar sedimentos durante maré vazante na parte NW da área, mas os vetores indicam transporte preferencial para SW, o que contraria todas as observações para a área em questão. Entre os programas testados, o GSTAST (figura 7) é o que melhor satisfaz as tendências esperadas. A iniciativa de Chang et al. (2001) de inserir um teste de significância para os vetores, bem como a geração de vetores adicionais empíricos que são utilizados no processo de filtragem, parece funcionar a contento neste caso em particular. Os vetores a leste da área refletem o transporte de material pelas ondas quebrando no banco da Galheta, os vetores centrais mostram o empilhamento da deriva de sul para norte sobre o delta de desembocadura e os vetores a oeste da área refletem a deflexão da deriva litorânea próximo à súbita queda na profundidade causada pela presença do delta.

É fato que as premissas sugeridas pelos autores para a aplicabilidade dos modelos são várias e, conseqüentemente, ambientes ideais são difíceis de serem encontrados na natureza. Não é objetivo deste trabalho criticar ou validar a aplicabilidade dos modelos existentes, mas sim, dentro de possibilidades reais, analisar qual dos programas testados apresenta melhores resultados no ambiente deposicional em questão.

\section{AGRADECIMENTOS}

Ao convênio APPA-CEM pelo apoio logístico e financeiro e à Capes pela bolsa de estudos. 
VEIGA, F. A. et al. Padrões de transporte de sedimentos baseado em três...

\section{REFERÊNCIAS}

ANGULO R.J. 1992. Geologia da planície costeira do estado do Paraná. Universidade de São Paulo - USP, São Paulo, Tese de Doutorado, 334p.

1993. Variações na configuração da linha de costa no

Paraná nas últimas quatro décadas. Boletim Paranaense de Geociências, Curitiba, 41:52-72.

1995. Feições deposicionais associadas às desembocaduras dos complexos estuarinos da costa paranaense. In: Congresso da Associação Brasileira de Estudos do Quaternário, 5, ABEQUA, Niterói, Resumos expandidos... 5864.

1999. Morphological characterization of the tidal deltas on the coast of the state of Paraná. Anais da Academia Brasileira de Ciências, 71:935-959.

BIGARELLA J.J. 1946. Contribuição ao estudo da planície litorânea do estado do Paraná. Arq. Biol. Tecnol., Curitiba, 4:1175.

BIGARELLA J.J., BECKER R.D., MATOS D.J. \& VERNER A. 1978. A Serra do Mar e a porção oriental do Estado do Paraná: um problema de segurança ambiental e nacional. Curitiba, Governo do Paraná/SEPL/ADEA, 249p.

CAMARGO M.G. 1999. SysGran para Windows: sistema de análises granulométricas. Pontal do Sul - PR.

CHANG Y.H., SCRIMSHAW M.D., LESTER J.N. 2001. A revised Grain-Size Trend Analysis program to define net sediment transport pathways. Computers \& Geosciences, 27:109-114.

FOLK R. L. \& WARD W. C. 1957. Brazos River Bay: Study of the significance of grain size parameters. Journal of Sedimentary Petrology, 27:3-27.

GAO S. 1996. A Fortran program for grain-size trend analysis to define net sediment transport pathways. Computers \& Geosciences, 22(4):449-452.

GAO S. \& COLLINS M., 1991. A critique of the Mclaren method for defining sediment transport paths-discussion. Journal of Sedimentary Petrology, 61:143-146.

1992. Net sediment transport patterns inferred from grain-size trends, based upon definition of transport vectors. Sedimentary Geology, 81:47-60.

1994. Analysis of grain size trends,for defining sediment transport pathway in marine environments. Journal of Coastal Research,10:70-78.

KNOPPERS B.A., BRANDINI F.P., THAMM C.A. 1987. Ecological studies in the bay of Paranaguá. II. Some physical and chemical characteristics. Nerítica, Curitiba, 2:1-36.

LAMOUR M.R. 2000. Dinâmica sedimentar do Canal da Galheta, via de acesso ao porto de Paranaguá - PR. Universidade Federal do Paraná, Curitiba, Dissertação de Mestrado, 100p.

LE ROUX J.P.1994a. A spreadsheet template for determining sediment transport vectors from grain-size parameters.
Computers \& Geosciences, 20(3):433-440.

LE ROUX J.P. 1994(b). An alternative approach to the identification of net sediment transport paths based on grainsize trends. Sedimentary Geology, 94:97-107.

LE ROUX J. P., O'BRIEN R.D., RIOS F., CISTERNAS M. 2002. Analysis of sediment transport paths using grain-size parameters. Computers \& Geosciences, 28:717-721.

MAACK R. 1968. Geografia física do Estado do Paraná. Curitiba, BADEP/UFPR/IBPT, 350p.

MARONE E. \& CAMARGO R. 1994. Marés meteorológicas no litoral do Estado do Paraná: o evento de 18 de agosto de 1993. Nerítica, Curitiba, 8:73-85.

MARTINS G.J. 2002. Dinâmica da zona de arrebentação e suas potenciais consequências no transporte de sedimentos na costa adjacente a desembocadura sul do complexo estuarino de Paranaguá. Universidade Federal do Paraná, Curitiba, Dissertação de Mestrado, 58p.

McCAMMON R.B. 1962. Efficiencies of percentile measurements for describing the mean size and sorting of sedimentary particles. Journal of Geology, 70:453-465.

McLAREN P. 1981. An interpretation of trends in grain size measures. Journal of Sedimentary Petrology, 51(2):611-624.

McLAREN P. \& BOWLES D. 1985. The effects of sediment transport on grain-size distributions. Journal of Sedimentary Petrology, 55(4):457-470.

NOERNBERG M. A. 2001. Processos morfodinâmicos no complexo estuarino de Paranaguá - Paraná - Brasil. Um estudo a partir de dados in situ e LandSat TM. Universidade Federal do Paraná, Curitiba, Tese de Doutorado, 180p.

PEDREROS R., HOWA H.L., MICHEL D. 1996. Application of grain size trend analysis for the determination of sediment transport pathways in intertidal areas. Marine Geology, 135: 35-49.

PORTOBRAS - EMPRESA DE PORTOS DO BRASIL S. A. 1983. Campanha de medições de ondas em Paranaguá-PR no período de 21/08/1982 a 21/01/1983. Rio de Janeiro, INPH - DIDELE, 90p.

SUGUIO K. 1973. Introdução à sedimentologia. São Paulo, Edgard Blucher/Edusp, 317p.

VEIGA F.A. \& ANGULO R.J. 2003. Sedimentologia da plataforma continental interna rasa na porção central da costa paranaense. Anais do IX Congresso da Associação Brasileira de Estudos do Quaternário. CD de trabalhos completos. Volume único.

VEIGA F.A., ANGULO R.J., MARONE E., BRANDINI F.P. 2004. Características sedimentológicas da plataforma continental interna rasa na porção central do litoral paranaense. Boletim Paranaense de Geociências, 55:67-75.

WATSON G.S. 1966. The statistics of orientation data. Journal of Geology, 74(2):786-797. 